\title{
Source position reconstruction and constraints on the galactic magnetic field from ultra-high energy cosmic rays
}

\author{
Geraldina Golup, Diego Harari, Silvia Mollerach and Esteban Roulet \\ CONICET and Centro Atómico Bariloche \\ Av. Bustillo 9500, 8400, S. C. de Bariloche, Argentina
}

\begin{abstract}
We study the possibility to reconstruct the position of ultra-high energy cosmic ray sources and some properties of the magnetic field along the line of sight towards them in the case that several events from the same source are detected. By considering an illustrative model for the galactic magnetic field, including both a regular and a turbulent component, we estimate the accuracy that can be achieved in the reconstruction. We analyse the effect of the experimental energy and angular resolutions on these results. We show that if about ten events with energies above 30 $\mathrm{EeV}$ are detected coming from the same source, it should be possible to reconstruct the source position with an accuracy of $0.5^{\circ}$ and the integral of the orthogonal component of the magnetic field along the line of sight with an accuracy of $0.6 \mu \mathrm{G} \mathrm{kpc} \mathrm{Z}^{-1}$ (with $\mathrm{Z}$ the electric charge of the particles).
\end{abstract}

Key words: High Energy Cosmic Rays

PACS: 98.70.Sa

\section{Introduction}

The recent discovery by the Pierre Auger Collaboration [1] that the arrival directions of the ultra-high energy cosmic rays are correlated with the nearby extragalactic matter distribution marks a first step in the cosmic ray astronomy era. Out of their 27 highest energy events, 20 correlate with the position of nearby active galactic nuclei (AGN), with two events near Cen A, our closest AGN. Previously, the combined data of AGASA, Haverah Park, Volcano Ranch and Yakutsk showed signals of clustering, including eight doublets and two triplets within $4^{\circ}$ angular separation [2]. It is to be expected that with increased statistics future data will contain evidence of multiplets, i.e. cosmic rays of different energies coming from the same point-like source. From the arrival direction and energy of multiplets one can extract not only the position of the source but also valuable information about the magnetic field along the line of sight towards it. In this work we analyse how to reconstruct this information considering the effect that energy and angular experimental resolutions have on the results and estimate the accuracy that can be achieved.

Despite the considerable observational efforts done, the magnetic field of the Galaxy and the extragalactic ones are still poorly known. This, together with the fact that the cosmic ray composition at ultra-high energies is also poorly known, makes it difficult to identify the cosmic ray sources, as it is not possible to predict the deflection of the cosmic ray trajectories in their way 
from the sources to the Earth. Propagation of charged particles through the galactic magnetic field has been studied in detail previously in many references. These studies are usually done backtracking antiparticles leaving the Earth with an initial direction pointing to the arrival direction of the cosmic ray particle [3, 4, 5, 6, 7, 8]. Alternatively, the forward tracking technique, consisting of tracking the trajectories of a large number of particles leaving the source and keeping the arrival directions of those particles that arrive to a given neighborhood of the Earth, has been used in [9]. Bisymmetric [3, 5, 6, 7, 8, 9] and axisymmetric [3, 4, 5, 8] magnetic field models have been considered, as well as a dipole [7, 8, 9] and toroidal components [7]. A turbulent magnetic field component was included in [7, 9]. These studies show that the magnitude and direction of the resulting deflections are strongly dependent on the details of the magnetic field model considered.

The observation of several events from the same source would open the possibility to actually measure the integral of the component of the magnetic field orthogonal to the cosmic ray trajectory, providing a new constraint to galactic magnetic field models, besides the possibility to locate more accurately the source position. Magnetic fields not only deflect the trajectories of charged particles but they can also amplify or demagnify the flux arriving from the source, modifying its spectrum. A detailed analysis of magnetic lensing effects can be found in refs. $[5,10,11,12]$.

In this paper we will use simulations of sets of events arriving to the Earth from randomly located sources in the sky after travelling through the galactic magnetic field, which we model using a regular and a turbulent component that aim to reproduce the general characteristics of the observational results. For each hypothetical source direction in the sky we consider several cosmic ray particles with different energies for which we compute the arrival directions to the Earth. The energies of the simulated events are generated using a power law at the source, which is then modified according to the lensing effects expected in each sky direction [5]. For each simulated set of events from a source we then reconstruct the original direction of the source and the integral of the orthogonal component of the magnetic field, comparing the results with the actual values to estimate the uncertainty of the method and the effect of the experimental error in the determination of the energy and the arrival direction.

The paper is structured as follows: in section II we describe the general known characteristics of the galactic magnetic field and review some magnetic lensing facts. Then, in section III, we explain the method used for the analysis and reconstruction and in section IV we present and discuss the accuracy that can be achieved in the reconstruction of the magnetic field integral and of the source position for realistic situations. Finally, section V is a summary of the results and contains our conclusions.

\section{Galactic Magnetic Field and Magnetic Lensing}

The present knowledge of the galactic magnetic field is far from complete. There are several observational methods for determining it: Zeeman splitting, polarized thermal emission from dust in clouds, polarization of starlight, synchrotron radio emission and Faraday rotation of polarized sources [13, 14]. When using rotation measures of galactic pulsars to constrain the three 
most widely used theoretical models for the magnetic field in the Galactic disk, namely the circular, the axisymmetric and the bisymmetric field models, none of them can consistently fit all the data, although the bisymmetric model appears to perform better than the others [15]. The observation of ultra-high energy cosmic ray multiplets could provide a new handle to probe the galactic magnetic field.

The galactic magnetic field has a large scale regular component and a turbulent component present on galactic scales. From the analysis of the polarization of starlight it is known that the local regular component is approximately parallel to the galactic plane and follows the local spiral arms [16]. The regular component has a local value of $B_{\text {reg }} \simeq 2 \mu \mathrm{G}$ according to Faraday rotation measures [17] (although this local value could be underestimated if the fluctuations in magnetic field and electron density were anticorrelated [18]). The local value of the regular field derived from synchrotron measurements is larger, $B_{\text {reg }} \simeq 4 \mu \mathrm{G}[14]$, but it could be overestimated due to the anisotropy of the turbulent magnetic field [18, 19, 20]. According to some models the regular magnetic field has reversals in direction between neighboring arms. From Faraday rotation data there is evidence that in the inner arms magnetic fields are counterclockwise when viewed from the North Galactic pole, while in the local region the fields in the interarm regions are clockwise [13]. Rotation measures also indicate that the magnetic field in the galactic halo appears to be antisymmetric with respect to the galactic plane and such a field could be produced by an A0 dynamo mode [13]. However, it is argued that the superposition of a disk field with even parity and a halo field with odd parity cannot be explained by classical dynamo theory [14].

The random component has a root mean square amplitude of $B_{r m s} \simeq(1-2) B_{\text {reg }}$ and a typical coherence length $L_{c} \simeq 100 \mathrm{pc}[21,22]$. The regular component, being coherent on scales much larger than $L_{c}$, produces the dominant effect on the deflection of high energy charged particles travelling through the Galaxy. In addition, the presence of extragalactic magnetic fields could also be relevant for the propagation of cosmic rays. However, their strength is very uncertain (and likely subdominant as regards the deflecting power) and we will not consider their effects here.

In this paper we consider an illustrative galactic field model that includes some of the observed features as field reversals and reproduce the local field strength. The regular component of the galactic magnetic field is modeled with a bisymmetric field with even symmetry (BSS-S) with structure and strength very similar to those used in [3] but smoothed out as described in [5]. In this model the galactic magnetic field reverses its sign between the arms of the Galaxy and the field is symmetric with respect to the Galaxy's mid-plane. The local value of the field is taken as $2 \mu \mathrm{G}$. For the dependence on $z$ a contribution coming from the galactic disk and another one from the halo are considered:

$$
\vec{B}_{r e g}(x, y, z)=\vec{B}_{r e g}(x, y, z=0)\left(\frac{1}{2 \cosh \left(z / z_{1}\right)}+\frac{1}{2 \cosh \left(z / z_{2}\right)}\right)
$$

with $z_{1}=0.3 \mathrm{kpc}$ and $z_{2}=4 \mathrm{kpc}$. These values of the parameters $z_{1}$ and $z_{2}$ lead to a similar dependence with the height above the galactic plane $z$ as that obtained using an exponential profile $e^{-|z| / z_{0}}$ with scale heights $z_{0}=1.2 \mathrm{kpc}$ for $|z|<0.5 \mathrm{kpc}$ and $z_{0}=4 \mathrm{kpc}$ for $|z|>0.5 \mathrm{kpc}$, as considered in [3], but the expression adopted in eq. 1 allows to avoid the presence of singular derivatives on the plane and when matching the disk and halo profiles. To model the turbulent component of the magnetic field we use a Gaussian random field with zero mean and root mean 


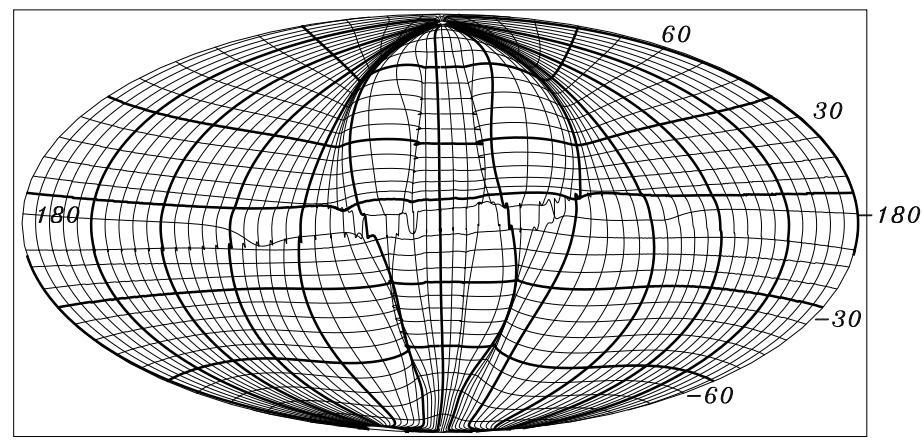

(a)

$B S S-S E / Z=20 \mathrm{EeV}$

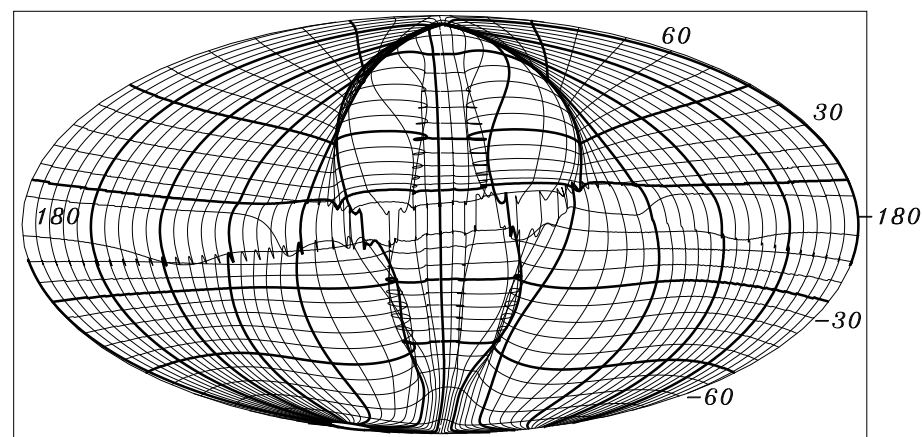

(b)

Figure 1: 'Sky sheets': directions of incoming cosmic rays in the halo that correspond to a regular grid of arrival directions at Earth, adopting the BSS-S magnetic field configuration, for particles with $E / Z=30 \mathrm{EeV}$ (a) and $20 \mathrm{EeV}$ (b). An Aitoff projection in galactic coordinates centered in the Galactic center is used.

square value $B_{r m s}$ equal to $2 \mu \mathrm{G}$. The coherence length $L_{c}$ adopted is $100 \mathrm{pc}$. For the dependence on $z$ of the turbulent component we consider a contribution coming from the disk and another one from the halo with the same scales as in the regular component.

The deflections caused by the magnetic field can lead to lensing phenomena, such as the energy dependent magnification and demagnification of the flux that modifies the energy spectrum of the source, or to the appearance of secondary images [5, 10, 11]. The magnitude of the flux amplification depends on the arrival direction, on the ratio between the energy and charge $E / Z$ of the cosmic ray and on the magnetic field model. The formation of multiple images and the flux magnification can be understood pictorially plotting for a regular grid of arrival directions at Earth the corresponding directions from which the particles arrived to the galactic halo. This is shown in Figure 1 for the model of the regular magnetic field configuration that is considered in this paper and for particles with $E / Z=30 \mathrm{EeV}$ (top panel) and $20 \mathrm{EeV}$ (bottom panel), where 1 $\mathrm{EeV} \equiv 10^{18} \mathrm{eV}$. One may picture this distorted image of the sky seen from the Earth as a sheet 
(the 'sky sheet') that can be stretched and folded. A source located in a fold of this sky sheet will have multiple images, i.e. cosmic rays of the same energy can arrive to the Earth from several different directions. Moreover, the flux coming from a source in a region where the sheet is stretched will appear demagnified while that from a source in a compressed region will appear magnified. As the sky sheet changes with the energy, the spectrum observed from a given source is different from the emitted one [5].

Magnetic lensing phenomena also appear for turbulent fields [11]. In this case, multiple images appear below a critical energy $E_{c}$ such that typical transverse displacements among different paths after travelling a distance $L$ in the turbulent field become of the order of the correlation length of the random magnetic field $\left(\delta_{r m s} \sim L_{c} / L\right)$. This critical energy is typically higher than the corresponding one for the regular magnetic field, but the folds produced by the random field near the critical energy are on a much smaller angular scale. For decreasing energies, the fraction of the sky covered with folds increases, however the magnification peaks become increasingly narrower and for $E<E_{c} / 3$ their integrated effect becomes less noticeable.

\section{Reconstruction of the source position and the magnetic field}

Charged particles of different energies coming from the same source suffer different deflections in their way through the Galaxy and are thus observed with different arrival directions. If deflections are small, the arrival direction $\vec{\theta}$ of a particle with energy $E$ is related to the source direction $\vec{\beta}$ by

$$
\vec{\beta}=\vec{\theta}+\frac{\vec{F}(\vec{\theta})}{E},
$$

where $\vec{F}$ is the integral along the line of sight of the perpendicular component of the magnetic field $\vec{B}$ times the charge $\mathrm{Ze}$ of the particle

$$
\vec{F}(\vec{\theta})=Z e \int_{0}^{L} \mathrm{~d} \vec{l} \times \vec{B}(\vec{l})
$$

To analyse the correlation between arrival direction and energy, we first fit in the tangent plane to the celestial sphere in the direction of the events (using coordinates $\alpha_{1}$ and $\alpha_{2}$ ) a straight line, $\alpha_{1}=a_{1}+a_{2} \alpha_{2}$, to the event coordinates in order to determine the direction of the deflection. This direction should coincide with the direction of $\vec{F}$. Then, we rotate to new coordinates $\left(\theta_{1}, \theta_{2}\right)$ along and orthogonal to the deflection direction. The procedure is illustrated in Figure 2.

If the deflections are small we can approximate $\vec{F}(\vec{\theta})$ as a constant value, lets say the value in the source direction $\vec{F}(\vec{\beta})$, and fitting a linear relation between $\theta_{1}$ and $1 / E$,

$$
\theta_{1}=\beta_{1}-\frac{F_{\theta_{1}}(\vec{\beta})}{E},
$$

we can obtain the component $\theta_{1}$ of the position of the source, $\beta_{1}$, and the component in the direction of the coordinate $\theta_{1}$ of the integral $\vec{F}$ evaluated at the position of the source, $F_{\theta_{1}}(\vec{\beta})$. The position of the source in these coordinates will be $\vec{\beta}=\left(\beta_{1}, 0\right)$ as the deflection is essentially along 


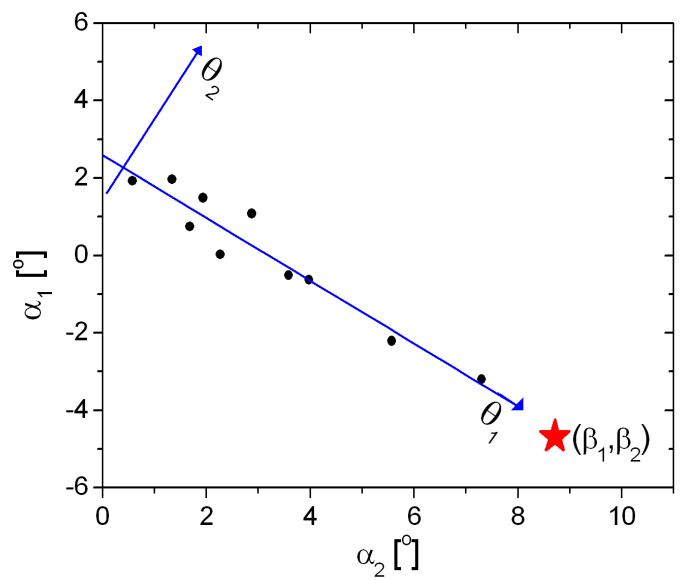

Figure 2: An example of the rotations performed. In the tangent plane to the celestial sphere in the direction of the events, coordinates $\left(\alpha_{1}, \alpha_{2}\right)$, we perform a linear fit to the event coordinates in order to determine the direction of the deflection. The new coordinates are named $\left(\theta_{1}, \theta_{2}\right)$, along and orthogonal to the deflection direction. In these new coordinates, the source position es $\left(\beta_{1}, \beta_{2}\right)$.

the coordinate $\theta_{1}$.

The fact that particles of different energies follow different paths along the galactic magnetic field has as a consequence that $\vec{F}(\vec{\theta})$ is not actually constant, so departures from the linear relation are expected, specially for the lower energy events, which follow more bent paths. In these cases the next term in the expansion can be relevant

$$
\beta_{1}=\theta_{1}+\frac{1}{E}\left[F_{\theta_{1}}(\vec{\beta})+\left.\frac{\partial F_{\theta_{1}}}{\partial \theta_{1}}\right|_{\vec{\beta}}\left(\theta_{1}-\beta_{1}\right)\right] .
$$

Assuming that $\left(1+\left.\frac{1}{E} \frac{\partial F_{\theta_{1}}}{\partial \theta_{1}}\right|_{\vec{\beta}}\right)^{-1} \simeq\left(1-\left.\frac{1}{E} \frac{\partial F_{\theta_{1}}}{\partial \theta_{1}}\right|_{\vec{\beta}}\right)$, one obtains

$$
\theta_{1} \simeq \beta_{1}-\frac{F_{\theta_{1}}(\vec{\beta})}{E}+\left.\frac{1}{E^{2}} F_{\theta_{1}}(\vec{\beta}) \frac{\partial F_{\theta_{1}}}{\partial \theta_{1}}\right|_{\vec{\beta}} .
$$

We will test here the accuracy that can be achieved in the reconstruction of the relevant parameters using this method. We work with simulations of protons coming from extragalactic point-like sources and propagating through a toy model of the galactic magnetic field that aims to reproduce the general characteristics of the observational results. These simulations are done backtracking antiprotons leaving the Earth up to a distance where the effect of the galactic magnetic field becomes negligible [5]. The initial direction of the backtracked antiproton (that will be associated to the arrival direction of protons of the same energy) is recursively adjusted till the final direction points to the source with accuracy better than $10^{-4}$ degrees when only the regular component of the galactic magnetic field is considered and better than $0.3^{\circ}$ when both the regular 
and turbulent components are considered.

We will consider a large number of source directions in order to characterize the deflections expected in different regions of the sky. For each source direction we simulate 10 events with energies randomly chosen between 30 and $300 \mathrm{EeV}$ following an $E^{-2}$ spectrum at the source and taking into account the magnification of each source flux as a function of the energy for that direction. We propagate them through the magnetic field, keeping track of the arrival direction to the Earth. In order to study the effect of the energy and angular resolution a random deflection of up to $1^{\circ}$ is added to the position and a random shift in energy of up to $10 \%$ is introduced. The reconstruction accuracy slightly depends on the particular realization of the experimental uncertainty, we thus present the results for the mean of 100 different realizations. When the random component of the galactic magnetic field is introduced the results also slightly depend on the particular realization of the field, we then present the results of 10 different realizations of the random field ${ }^{1}$.

In each case we perform a linear and a quadratic fit to the relation $\theta_{1}$ vs. $1 / E$ for the events and obtain the values of $F_{\theta_{1}}(\vec{\beta})$ and the position of the source $\left(\beta_{1}, \beta_{2}\right)$. The actual value of $\vec{F}(\vec{\beta})$ is calculated with a numerical integration along a straight path. By comparing the reconstructed values of $F_{\theta_{1}}(\vec{\beta})$ and $\vec{\beta}$ with the actual ones we can estimate the accuracy of the reconstruction. We also analyse the angle between $\vec{F}(\vec{\beta})$ and the direction of the deflection obtained from the fit to the data, $\epsilon$, which measures the accuracy in the reconstruction of the direction of $\vec{F}(\vec{\beta})$.

In Figure 3 we show the source position and the cosmic ray arrival directions for 100 randomly selected directions in the sky and in Figure 4 we present the distribution of $F_{\theta_{1}}(\vec{\beta})$ for these positions. As one can see from these figures, the magnitude of the deflection depends on the region of the sky where the source is located and has a mean value $\left\langle F_{\theta_{1}}\right\rangle=2^{\circ} 100 \mathrm{EeV}\left(1^{\circ} 100\right.$ $\mathrm{EeV} \approx 1.9 e \mu \mathrm{G} \mathrm{kpc})$. Regarding the magnification, at $30 \mathrm{EeV}$ we find that 19 sources have an amplification smaller than 0.5 and 5 sources have an amplification larger than 1.5. Moreover, 7 sources have a magnification peak with magnification larger than 3 at some energy above the 30 $\mathrm{EeV}$ threshold. In these peaks, the amplification of the source remains larger than 1.5 for a range of energies of about 10 to $20 \mathrm{EeV}$.

To illustrate the reconstruction method and its accuracy we first present in detail the results for three particular source directions which are representative of different types of deflections. The reconstruction accuracy for the different quantities are summarized in Table 1. The values presented are median values, i.e. 50\% of the realizations of the experimental uncertainties and of the turbulent field have equal or better accuracy. For each case we first present the results without taking into account any experimental uncertainty. Thus, these results represent the uncertainties inherent to the method, that are due to the departures of the actual deflections from the linear or quadratic relations as a function of $1 / E$. We then introduce the experimental uncertainty in the

\footnotetext{
${ }^{1}$ In a realistic observational situation, the events from one source could be surrounded by background events from other sources, in which case one can select the candidate source events using their filamentary structure [23] and the expected high degree of correlation between their deflections and $1 / E$. A particularly useful tool for this purpose is given by the correlation coefficient $C\left(\theta_{1}, 1 / E\right)=\operatorname{Cov}\left(\theta_{1}, 1 / E\right) / \sqrt{\operatorname{Var}\left(\theta_{1}\right) \operatorname{Var}(1 / E)}$. Requiring this coefficient to be sufficiently close to unity for the selected events would ensure their high degree of correlation and hence a suppressed contribution from background events.
} 


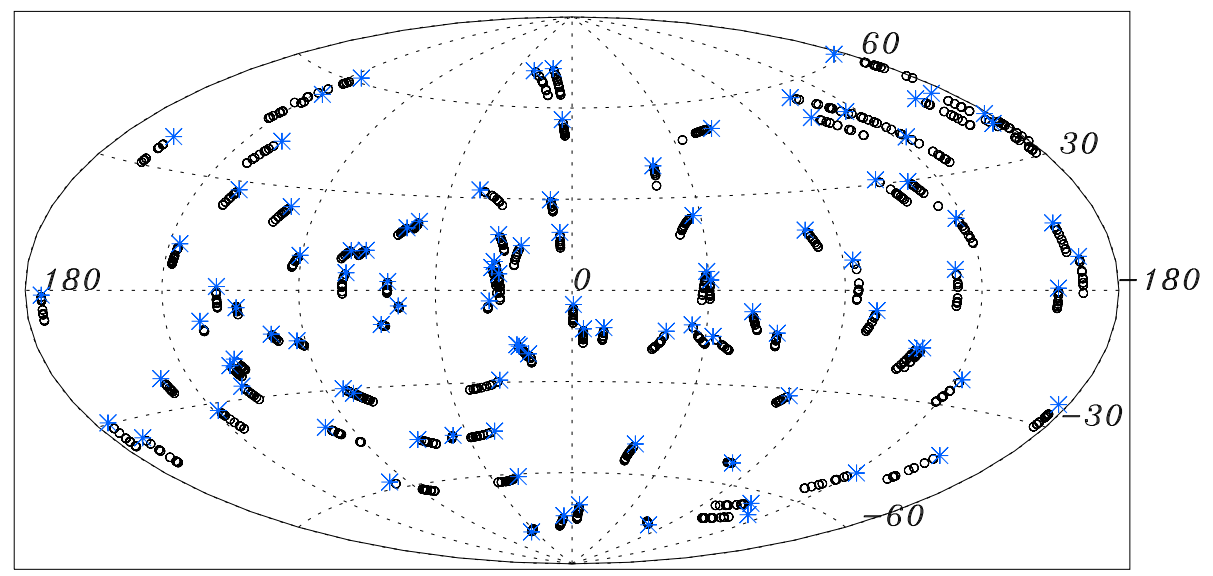

Figure 3: One hundred sources with randomly selected directions in the sky (asterisks) and ten cosmic rays with $E / Z \geq 30$ $\mathrm{EeV}$ coming from each of these sources (circles) in an Aitoff projection of the celestial sphere in galactic coordinates.

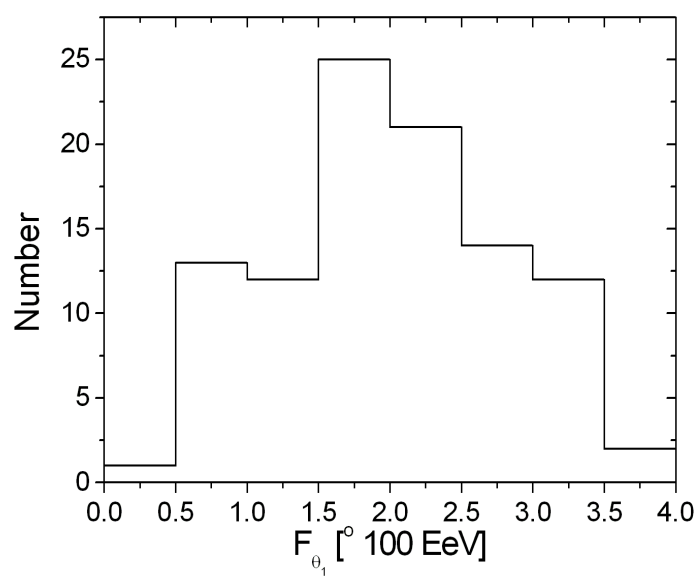

Figure 4: Distribution of $F_{\theta_{1}}(\vec{\beta})$ obtained with numerical integration for the one hundred sources considered.

angle and the energy so that it is possible to see its effect on the reconstruction accuracy.

In the first example the source is located in galactic coordinates $(l, b)=\left(220^{\circ},-60^{\circ}\right)$, corresponding to a region of the sky with a large deflection $\left(F_{\theta_{1}}=3.3^{\circ} 100 \mathrm{EeV}\right)$. We see from Table 1 that the value of $F_{\theta_{1}}$ is well reconstructed with the linear fit with median relative errors smaller than $10 \%$. The quadratic fit gives even more accurate results both for the source position and for $F_{\theta_{1}}$ when no measurement uncertainties are introduced. When the experimental uncertainties (of the magnitude discussed above) are introduced, the mean reconstructed value of $F_{\theta_{1}}$, 


\begin{tabular}{|c|c|c|c|c|c|}
\hline & $\begin{array}{c}\Delta F_{\theta_{1}} \\
{\left[{ }^{\circ} 100 \mathrm{EeV}\right]}\end{array}$ & $\begin{array}{c}\epsilon \\
{\left[{ }^{\circ}\right]}\end{array}$ & $\begin{array}{c}\Delta \beta_{1} \\
\left.{ }^{\circ}\right]\end{array}$ & $\begin{array}{c}\Delta \beta_{2} \\
{\left[{ }^{\circ}\right]}\end{array}$ & $\begin{array}{c}|\Delta \vec{\beta}| \\
{\left[{ }^{\circ}\right]}\end{array}$ \\
\hline \multicolumn{6}{|c|}{ Example 1: $(l, b)=\left(220^{\circ},-60^{\circ}\right)$} \\
\hline Regular, linear fit & 0.21 & 1.0 & 0.2 & 0.04 & 0.2 \\
\hline Regular, quadratic fit & 0.11 & 1.0 & 0.04 & 0.04 & 0.05 \\
\hline Reg. + error, linear fit & 0.31 & 1.7 & 0.5 & 0.3 & 0.6 \\
\hline Reg. + error, quadratic fit & 0.75 & 1.7 & 0.6 & 0.3 & 0.7 \\
\hline Turbulent, linear fit & 0.16 & 1.0 & 0.1 & 0.07 & 0.2 \\
\hline Turbulent, quadratic fit & 0.22 & 1.0 & 0.1 & 0.07 & 0.2 \\
\hline Turb. + error, linear fit & 0.28 & 2.3 & 0.4 & 0.3 & 0.5 \\
\hline Turb. + error, quadratic fit & 0.95 & 2.3 & 0.6 & 0.3 & 0.8 \\
\hline \multicolumn{6}{|l|}{ Example 2: $(l, b)=\left(55^{\circ}, 20^{\circ}\right)$} \\
\hline Regular, linear fit & 0.28 & 8.6 & 0.2 & 0.1 & 0.3 \\
\hline Regular, quadratic fit & 0.03 & 8.6 & 0.01 & 0.1 & 0.1 \\
\hline Reg. + error, linear fit & 0.25 & 21.0 & 0.3 & 0.4 & 0.6 \\
\hline Reg. + error, quadratic fit & 0.49 & 21.0 & 0.4 & 0.4 & 0.7 \\
\hline Turbulent, linear fit & 0.27 & 5.2 & 0.3 & 0.1 & 0.3 \\
\hline Turbulent, quadratic fit & 0.18 & 5.2 & 0.1 & 0.1 & 0.2 \\
\hline Turb. + error, linear fit & 0.27 & 19.1 & 0.4 & 0.6 & 0.8 \\
\hline Turb. + error, quadratic fit & 0.64 & 19.1 & 0.5 & 0.6 & 0.9 \\
\hline \multicolumn{6}{|l|}{ Example 3: $(l, b)=\left(20^{\circ},-40^{\circ}\right)$} \\
\hline Regular, linear fit & 0.35 & 10.2 & 0.4 & 0.02 & 0.4 \\
\hline Regular, quadratic fit & 0.24 & 10.2 & 0.07 & 0.02 & 0.07 \\
\hline Reg. + error, linear fit & 0.42 & 16.1 & 0.5 & 0.3 & 0.6 \\
\hline Reg. + error, quadratic fit & 0.42 & 16.1 & 0.4 & 0.3 & 0.6 \\
\hline Turbulent, linear fit & 0.36 & 7.0 & 0.5 & 0.09 & 0.5 \\
\hline Turbulent, quadratic fit & 0.28 & 7.0 & 0.08 & 0.09 & 0.1 \\
\hline Turb. + error, linear fit & 0.37 & 14.4 & 0.4 & 0.3 & 0.5 \\
\hline Turb. + error, quadratic fit & 0.47 & 14.4 & 0.4 & 0.3 & 0.6 \\
\hline
\end{tabular}

Table 1: Results for the three examples: difference between the reconstructed and the true value of $F_{\theta_{1}}, \Delta F_{\theta_{1}}$, of the direction of $\vec{F}$, angle $\epsilon$, and of the position of the source $\left(\beta_{1}, \beta_{2}\right)$. The corresponding true values of $F_{\theta_{1}}$ for each example are: $3.3^{\circ} 100 \mathrm{EeV}, 0.7^{\circ} 100 \mathrm{EeV}$ and $1.8^{\circ} 100 \mathrm{EeV}\left(1^{\circ} 100 \mathrm{EeV} \approx 1.9\right.$ e $\left.\mu \mathrm{G} \mathrm{kpc}\right)$. 
$\left\langle F_{\theta_{1}}\right\rangle=(3.0 \pm 0.4)^{\circ} 100 \mathrm{EeV}$, agrees within the error with the true value, $F_{\theta_{1}}=3.3^{\circ} 100 \mathrm{EeV}$, in the linear fit case. The quadratic fit leads to a significantly larger uncertainty in the determination of $F_{\theta_{1}},\left\langle F_{\theta_{1}}\right\rangle=(3.8 \pm 2)^{\circ} 100 \mathrm{EeV}$, that also encompasses the true value of $F_{\theta_{1}}$. On the other hand, the mean fitted value for the quadratic term, $\left\langle\left. F_{\theta_{1}}(\vec{\beta}) \frac{\partial F_{\theta_{1}}}{\partial \theta_{1}}\right|_{\vec{\beta}}\right\rangle=(0.3 \pm 0.4)^{\circ}(100 \mathrm{EeV})^{2}$ is compatible with zero. We see that performing a quadratic fit does not lead to an improvement of the reconstruction in this case for the values of the experimental uncertainties considered, as the addition of an extra parameter just lead to a larger uncertainty in the determination of $F_{\theta_{1}}$. The position of the source and the direction of $\vec{F}$ are also well reconstructed in all cases. The quadratic fit also leads to an increased uncertainty in $\beta_{1}$ with respect to the linear fit. In addition, the turbulent component does not have much effect in any result because the regular component gives the dominant contribution to the deflections. In Figure 5, the deflection as a function of $1 / E$ is portrayed for the four situations studied in this example.

For the second example, we consider a source located at $(l, b)=\left(55^{\circ}, 20^{\circ}\right)$, corresponding to a region with a small deflection $\left(F_{\theta_{1}}=0.7^{\circ} 100 \mathrm{EeV}\right)$. The error in the value of $F_{\theta_{1}}$ is comparable to that of the previous example (though the relative error is considerably larger, as $F_{\theta_{1}}$ is smaller) and the position of the source is well reconstructed, as it is shown in Table I. Both the mean reconstructed values of $F_{\theta_{1}}\left(\left\langle F_{\theta_{1}}\right\rangle=(0.9 \pm 0.4)^{\circ} 100 \mathrm{EeV}\right)$ and the source coordinate $\beta_{1}$ coincide within the error of the fit with the true values. The direction of $\vec{F}$ has a poorer reconstruction than in the previous example. This happens because as the deflection in this region of the sky is small, there is a smaller lever arm between the highest and the smallest energy events to fix the deflection direction and the uncertainties introduced in the angle are relatively more significant. Although the quadratic fit gives a better reconstruction of $F_{\theta_{1}}$ when measurement uncertainties are neglected, it is less accurate when adding errors as in the previous example. The turbulent component does not introduce substantial changes either in this case.

For the third example we consider a source at $(l, b)=\left(20^{\circ},-40^{\circ}\right)$, that corresponds to a region with deflections close to the mean $\left(F_{\theta_{1}}=1.8^{\circ} 100 \mathrm{EeV}\right)$ but near to a fold of the sky, as opposed to the other examples that were located in regions where the sky sheet does not suffer much deformation when lowering the energy of the incoming particles. For the linear fit, the mean reconstructed value of $F_{\theta_{1}},\left\langle F_{\theta_{1}}\right\rangle=(1.4 \pm 0.4)^{\circ} 100 \mathrm{EeV}$, coincides within the error with the true value. The median difference between the value of $F_{\theta_{1}}$ found and the real one is $23 \%$ and in the direction of $\vec{F}$ is $16.1^{\circ}$. The reconstruction without introducing the experimental errors is less accurate than in the first case because close to the folds the departures from the linear (and quadratic) approximation for the deflection as a function of $1 / E$ are larger. The position of the source is reconstructed with a median accuracy of $0.6^{\circ}$; and the quadratic fit behavior is similar to that in the previous examples.

If a random field with $B_{r m s}=4 \mu \mathrm{G}$ instead of $B_{r m s}=2 \mu \mathrm{G}$ is considered, multiple images due to the turbulent component can appear above $30 \mathrm{EeV}$ in the second example (in about half of the different realizations). However, the accuracy of the reconstruction is not much affected by the turbulent component also for this larger amplitude of the random field. 


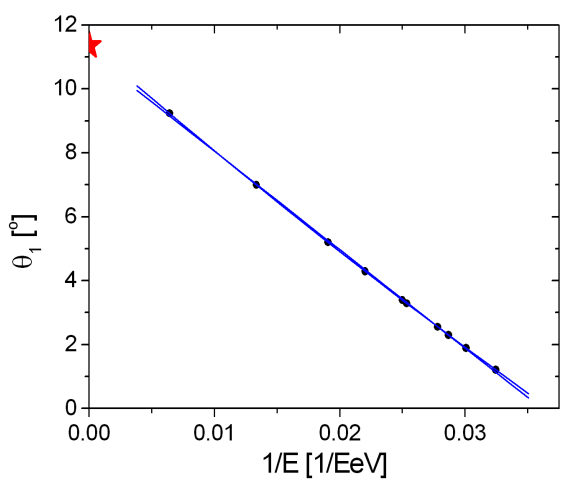

(a)

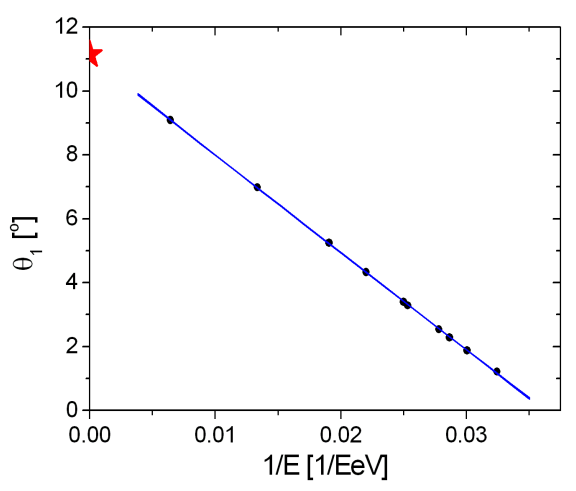

(c)

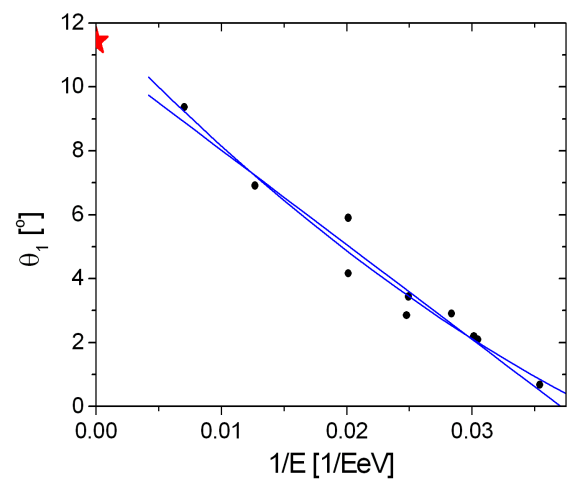

(b)

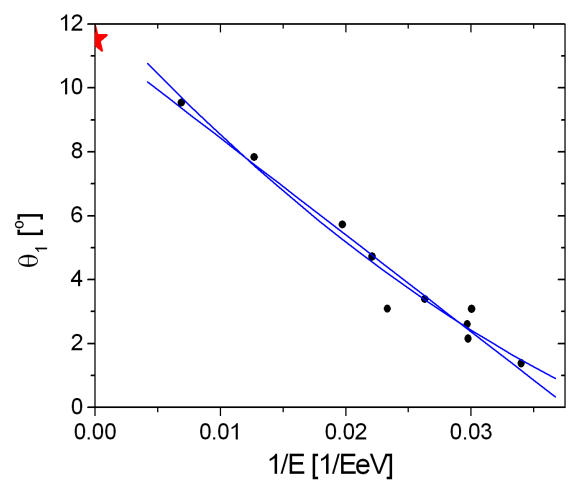

(d)

Figure 5: Deflection as a function of $1 / E$ for a source located at $(l, b)=\left(220^{\circ},-60^{\circ}\right)$ considering (a) magnetic field with a regular component, (b) regular component plus measurement uncertainties in energy and position, (c) regular and turbulent magnetic field, (d) regular and turbulent component plus measurement uncertainties in energy and position. The star indicates the true position of the source.

\section{Results}

In order to study the uncertainties in the reconstruction of $\vec{F}$ and in the position of the source we randomly select the location of 100 sources and perform the analysis described in the previous section to each one of them. We present the results for only the regular magnetic field component as the effects of the turbulent field on the accuracy of the reconstruction are small as exemplified in the previous section. The position of the sources are plotted in Figure 3 together with 10 cosmic rays arrival directions associated to each one, corresponding to the different cosmic ray energies considered. The results for the accuracy of the reconstruction are presented in Table II where the median of the difference between the reconstructed and the true value of $F_{\theta_{1}}$, $\Delta F_{\theta_{1}}$, of the angle defining the direction of $\vec{F}, \epsilon$, and of the position of the source, $\left(\Delta \beta_{1}, \Delta \beta_{2}\right)$, are quoted. The histograms for the full distribution for the 100 source positions and 100 realizations 
of the measurement uncertainties are plotted in Figures 6, 7 and 8.

\begin{tabular}{lccccc}
\hline \hline & $\begin{array}{c}\Delta F_{\theta_{1}} \\
{\left[{ }^{\circ} 100 \mathrm{EeV}\right]}\end{array}$ & $\begin{array}{c}\epsilon \\
\left.{ }^{\circ}\right]\end{array}$ & $\begin{array}{c}\Delta \beta_{1} \\
{\left[{ }^{\circ}\right]}\end{array}$ & $\begin{array}{c}\Delta \beta_{2} \\
{\left[{ }^{\circ}\right]}\end{array}$ & $|\Delta \vec{\beta}|$ \\
& & & & & \\
\hline & 0.25 & 3.9 & 0.2 & 0.09 & 0.2 \\
Regular, linear fit & 0.05 & 3.9 & 0.02 & 0.09 & 0.09 \\
Regular, quadratic fit & 0.32 & 5.8 & 0.3 & 0.3 & 0.5 \\
Reg. +error, linear fit & 0.65 & 5.8 & 0.5 & 0.3 & 0.6 \\
Reg. +error, quadratic fit & & & & & \\
\hline \hline
\end{tabular}

Table 2: Results for 100 sources with randomly selected positions. Median of the difference between the reconstructed and the true value of $F_{\theta_{1}}, \Delta F_{\theta_{1}}$, of the direction of $\vec{F}$, angle $\epsilon$, and of the position of the source $\vec{\beta}=\left(\beta_{1}, \beta_{2}\right), \Delta \beta_{1}, \Delta \beta_{2}$, $|\Delta \vec{\beta}|$, for the linear and the quadratic fits.

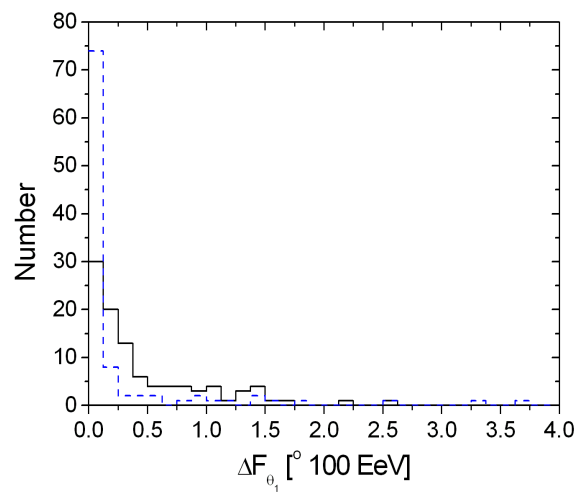

(a)

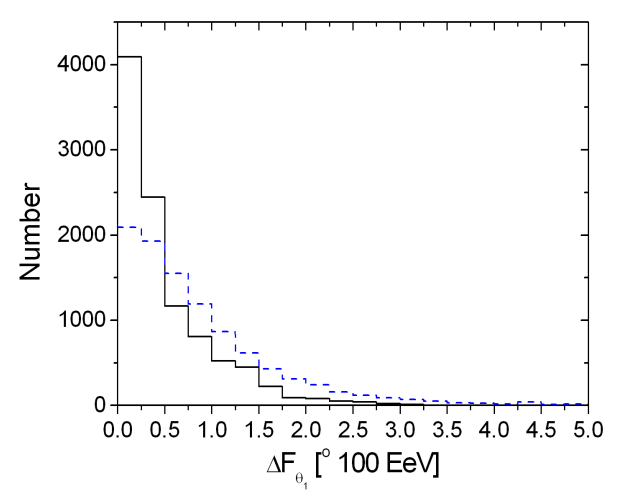

(b)

Figure 6: Histograms of the difference between the reconstructed and the true value of $F_{\theta_{1}}, \Delta F_{\theta_{1}}$, for the 100 source positions considering only a regular magnetic field without (a) and with (b) measurement uncertainties (for the 100 different realizations of the angle and energy uncertainties). Solid lines correspond to the linear fit and dashed lines to the quadratic fit results.

Applying a linear fit the reconstruction of $F_{\theta_{1}}$ is achieved with median difference between the reconstructed and the true value equal to $0.25^{\circ} 100 \mathrm{EeV}$ and $0.32^{\circ} 100 \mathrm{EeV}$ without and with measurement uncertainties respectively (i.e. $50 \%$ of the realizations have errors smaller than these values). On the other hand, in $57 \%$ of the cases the true value of $F_{\theta_{1}}$ lies within the error bar of the reconstructed value for the linear fit (in $90 \%$ of the cases it lies within 2.8 times the error bar). The corresponding median errors in the direction of $\vec{F}$ in these two cases are $3.9^{\circ}$ and $5.8^{\circ}$ respectively.

When comparing $\Delta F_{\theta_{1}}$ obtained with the linear fit and the one with the quadratic fit, the 


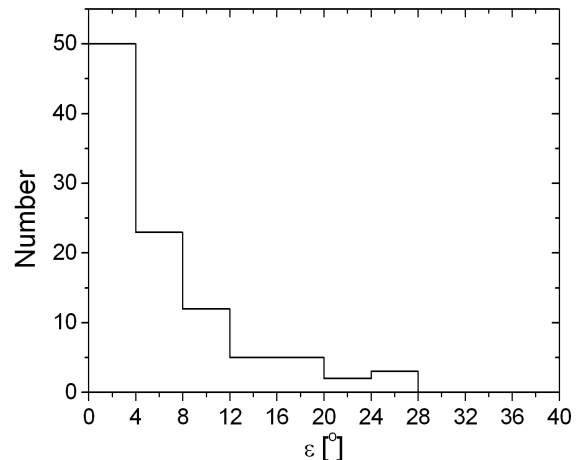

(a)

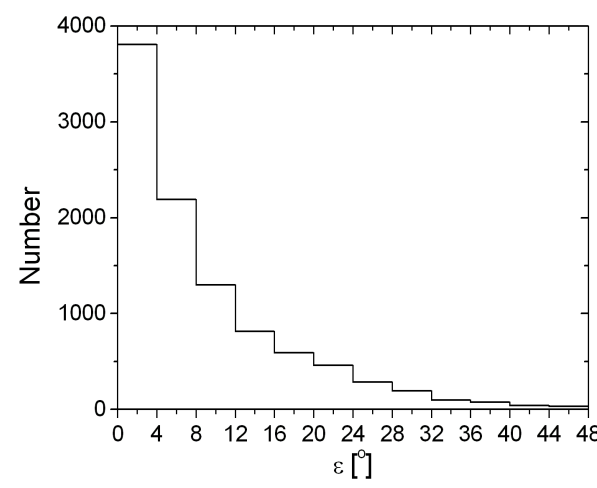

(b)

Figure 7: Histograms of the difference between the reconstructed and the true value of the direction of $\vec{F}, \epsilon$, for the 100 source positions considering only a regular magnetic field without (a) and with (b) measurement uncertainties.

latter is more accurate when no measurement uncertainties are introduced. However, when experimental errors of the magnitude considered are taken into account, the quadratic fit has larger uncertainties. This behavior is shown clearly in Figure 6 and in Table II where the median difference between the reconstructed values and the true values of $F_{\theta_{1}}$ for the quadratic fit grows from $0.05^{\circ} 100 \mathrm{EeV}$ to $0.65^{\circ} 100 \mathrm{EeV}$ when adding the measurement errors. Improving the experimental resolution, the accuracy of the quadratic fit improves, as it is shown in Figure 9. However, only an experiment with very good energy and angular resolution (for example $0.3^{\circ}$ in angle and 5\% in energy) would have a better accuracy by using the quadratic fit instead of the linear one for a statistics of events comparable to the one considered here.

The results for the reconstruction of the source direction are shown in Figure 8. Using the linear fit the position of the source is obtained with a median error of $0.2^{\circ}$ without introducing the experimental uncertainties and of $0.5^{\circ}$ when they are included. Once again, the median error applying a quadratic fit is lower, being $|\Delta \vec{\beta}|=0.09^{\circ}$ when no uncertainties are included, but when considering an experiment with an angular resolution of $1^{\circ}$ and an energy uncertainty of $10 \%$, rises to $|\Delta \vec{\beta}|=0.6^{\circ}$. In $85 \%$ of the cases the true source position is within the error bar of the reconstructed direction in the linear fit case.

The fact that the quadratic fit reconstruction accuracy becomes worse than the linear fit one when the measurement errors are considered can be understood as follows. The mean value of the quadratic term contribution to the deflection in Eq. (5) for the 100 source directions is $\left\langle\left. F_{\theta_{1}}(\vec{\beta}) \frac{\partial F_{\theta_{1}}}{\partial \theta_{1}}\right|_{\vec{\beta}}\right\rangle=0.12^{\circ}(100 \mathrm{EeV})^{2}$. Thus the expected mean departure from the approximately linear deflection is smaller than the angular resolution considered $\left(1^{\circ}\right)$ up to energies around 35 $\mathrm{EeV}$. Therefore, in most of the cases the quadratic deflection term is smaller than the angular accuracy and the addition of an extra parameter to the fit leads to a worse determination of the linear term. Only for energy thresholds significantly below $35 \mathrm{EeV}$ would the quadratic fit give more accurate results for the magnetic field model considered. 


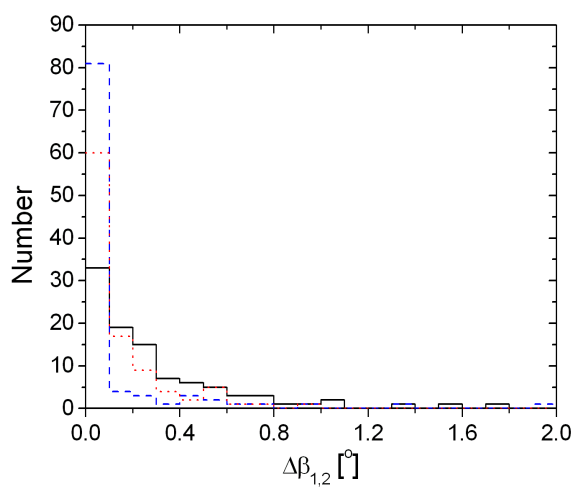

(a)

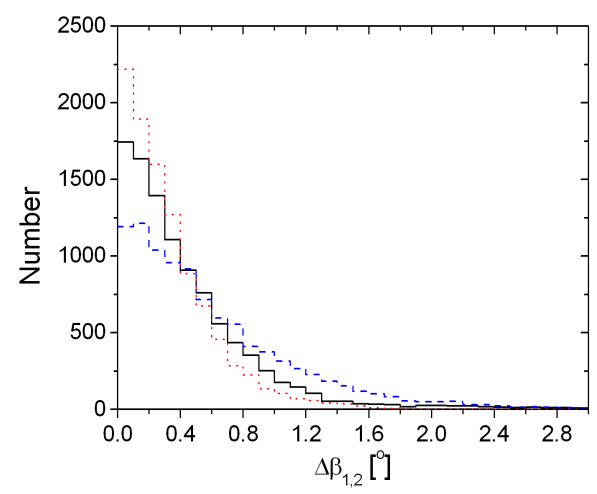

(b)

Figure 8: Histograms of the difference between the reconstructed and the true value of the position of the source $\left(\Delta \beta_{1}, \Delta \beta_{2}\right)$, for the 100 source positions considering only a regular magnetic field without (a) and with (b) measurement uncertainties. Solid lines correspond to the uncertainty in $\beta_{1}$ obtained with the linear fit, dashed lines to $\Delta \beta_{1}$ obtained with the quadratic fit and dotted lines to the uncertainty in $\beta_{2}$.

Regarding magnetic lensing effects, from the 100 randomly selected sources, 20 of the positions are crossed by a fold at energies above $30 \mathrm{EeV}$ and this fact affects the reconstruction of $\vec{F}$ as it is illustrated in the third example discussed in the previous section. Out of the 20, in 16 cases the fold crosses the position of the source at energies between 30 and $40 \mathrm{EeV}$.

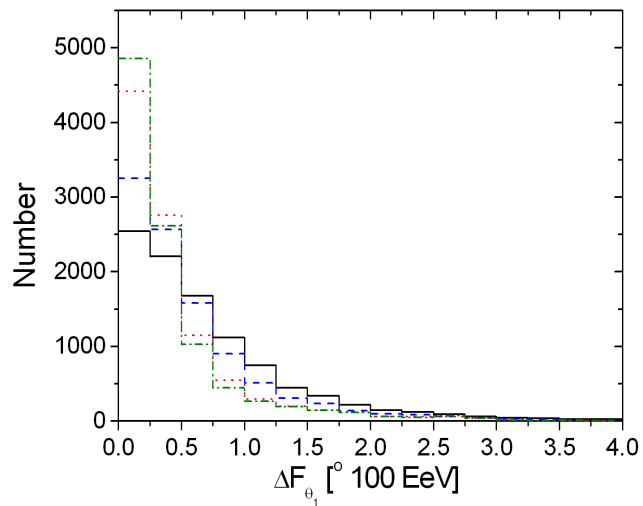

Figure 9: Histograms of the difference between the reconstructed and the true value of $F_{\theta_{1}}, \Delta F_{\theta_{1}}$, obtained with a quadratic fit, considering a regular magnetic field with measurement uncertainties. Solid lines correspond to an angular resolution of $0.8^{\circ}$ and an energy uncertainty of $8 \%$, dashed lines to $0.6^{\circ}$ and $6 \%$, dotted to $0.4^{\circ}$ and $4 \%$ and dash-dot lines to $0.3^{\circ}$ and $5 \%$. 
If one includes a turbulent component of the galactic magnetic field, the results are similar except for some of the sources near the galactic plane for which the cosmic ray trajectories traverse a larger region with turbulent magnetic field, leading to multiple images at energies above the 30 $\mathrm{EeV}$ threshold considered. This happens for 14 of the source locations considered. To illustrate this point we plot in Figure 10 the deflection as a function of $1 / E$ for two source positions in the cases in which only the regular component of the magnetic field is considered and considering both the regular and the turbulent components. The left panel corresponds to a source located at $(l, b)=\left(223.6^{\circ},-50^{\circ}\right)$ while the right panel corresponds to a source near the galactic plane at $(l, b)=\left(117.1^{\circ}, 1.1^{\circ}\right)$. In the first case, exemplifying sources far from the galactic plane, the turbulent component only produces small departures of the arrival direction of cosmic rays from that resulting from the regular component alone. The source near the galactic plane (right panel) shows a similar behaviour for energies larger than $75 \mathrm{EeV}$, but below this energy multiple images appear close to the principal one. The deflections in the energy range considered are however small and the resulting angular spread in the event directions is not much larger than the angular resolution considered. If one compares the accuracy of the linear fit reconstruction for the case of 10 events simulated in the magnetic field with regular and turbulent components and for the case considering only the regular component, but including the experimental uncertainties, the results are similar with the exception of the accuracy in the reconstruction of the direction of $\vec{F}$, for which the median of $\epsilon$ grows from $5.8^{\circ}$ to $12.3^{\circ}$. This is due to the fact that the turbulent field has no preferential direction and hence adds to the integral along the path of the charged particle for both $F_{\theta_{1}}$ and $F_{\theta_{2}}$. However, out of the 14 source positions for which multiple images above $30 \mathrm{EeV}$ were obtained, the one just considered is the source with the lowest value of $|\vec{F}|$ and therefore corresponds to the worst case.

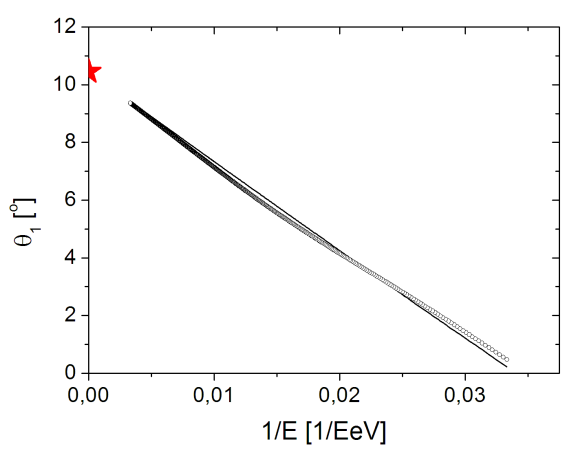

(a)

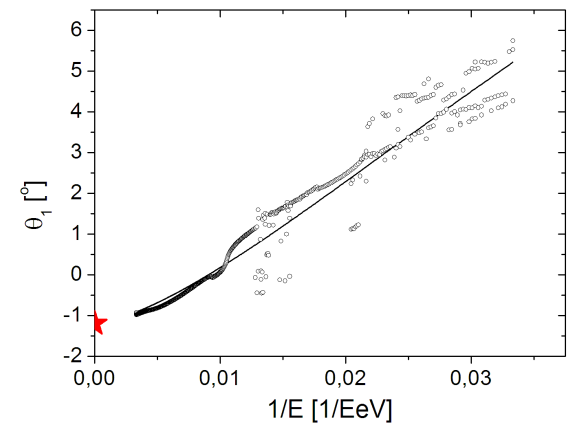

(b)

Figure 10: Deflection as a function of $1 / E$ for two sources: (a) is located at $(l, b)=\left(223.6^{\circ},-50^{\circ}\right)$ and (b) is near the galactic plane at $(l, b)=\left(117.1^{\circ}, 1.1^{\circ}\right)$. The solid line correspond to the deflection caused considering only a regular component of the galactic magnetic field and empty circles correspond to the deflection caused by both a regular and a turbulent component. In (b) some of the secondary images of the source due to the turbulent component are shown. The star indicates the true position of the source.

One may wonder if once a set of events from a source is identified it may be more convenient to use only a subset of the most energetic events for the reconstruction, as the linear relation is more accurate at high energies. In fact, when no measurement uncertainties are taken into 
account, the reconstruction of $\vec{F}$ and the position of the source improves when using only the most energetic events. For instance, if we consider the 5 most energetic events out of the 10 we simulated for each source, corresponding approximately to adopting an energy threshold of 50 $\mathrm{EeV}$, the linear fit gives in $50 \%$ of the examples accuracies better than: $\Delta F_{\theta_{1}}=0.16^{\circ} 100 \mathrm{EeV}$, $\epsilon=2.2^{\circ}$ and $|\Delta \vec{\beta}|=0.09^{\circ}$. Therefore, the reconstruction accuracy is similar as when applying the quadratic fit considering all the events. However, when experimental uncertainties are considered, the median accuracies of the reconstruction applying a linear fit to the 5 most energetic events rise to: $\Delta F_{\theta_{1}}=0.46^{\circ} 100 \mathrm{EeV}, \epsilon=10.5^{\circ}$ and $|\Delta \vec{\beta}|=0.7^{\circ}$, and we see then that it is best to use all the events.

Also one may wonder if it would be preferable to consider a lower threshold for the energy, for example $20 \mathrm{EeV}$ instead of $30 \mathrm{EeV}$, allowing to gain more statistics. However, the linear fit reconstruction accuracy is not improved because the departures from the linear relation between the deflection and $1 / E$ are more relevant. For instance, if we select 16 events above $20 \mathrm{EeV}$ and take into account the experimental resolution, the accuracy obtained applying the linear fit is in $50 \%$ of the cases better than: $\Delta F_{\theta_{1}}=0.41^{\circ} 100 \mathrm{EeV}, \epsilon=6.0^{\circ}$ and $|\Delta \vec{\beta}|=0.5^{\circ}$. The accuracy of the reconstruction is comparable using the linear and quadratic fit for this energy threshold.

Another question is how the accuracy of the reconstruction depends on the number of events that are detected from a source. If instead of 10 events, as considered in the previous analysis, only 5 events above $30 \mathrm{EeV}$ were detected, the reconstruction accuracy does not change appreciably when measurement uncertainties are not introduced, but it moderately worsens when uncertainties are taken into account, becoming: $\Delta F_{\theta_{1}}=0.37^{\circ} 100 \mathrm{EeV}, \epsilon=7.1^{\circ}$ and $|\Delta \vec{\beta}|=0.6^{\circ}$.

Finally, future satellite experiments such as JEM-EUSO will have larger aperture, gathering hence a larger number of events, but will also have worse angular and energy resolution than ground based experiments. To study this difference, we consider an experiment with an angular resolution of $2^{\circ}$ and an energy uncertainty of $20 \%$ and multiplets of 20 events above $50 \mathrm{EeV}$. The median accuracy of the reconstruction for the 100 source directions considered are in this case: $\Delta F_{\theta_{1}}=0.45^{\circ} 100 \mathrm{EeV}, \epsilon=12.6^{\circ}$ and $|\Delta \vec{\beta}|=0.7^{\circ}$. Therefore, for the resolution and number of events considered, the accuracy would be worse than for the case of multiplets of 10 events above $30 \mathrm{EeV}$ with the resolution associated to ground based experiments.

\section{Conclusions}

We have studied the accuracy with which the position of the source and the integral of the orthogonal component of the magnetic field can be reconstructed in case that several events from the same source are detected. We have used simulated sets of events arriving to the Earth from randomly located sources in the sky after travelling through the galactic magnetic field that we model with a regular and a turbulent component. Although the amplitude and the direction of the deflections depend on the model considered, the sets of simulated events provide a sample of the realistic types of deflections that can be expected. Reconstruction using a linear and a quadratic fit to the relation between the events position and $1 / E$ has been analysed. For the magnetic field model considered and the reference value of 10 detected events with energy above $30 \mathrm{EeV}$, the median errors of the reconstruction of $F_{\theta_{1}}$ applying a linear fit are $0.25^{\circ} 100 \mathrm{EeV}$ when no experimental uncertainty is introduced and $0.32^{\circ} 100 \mathrm{EeV}$ when a $1^{\circ}$ uncertainty in the position and 
a $10 \%$ uncertainty in the energy are considered $\left(1^{\circ} 100 \mathrm{EeV} \approx 1.9 e \mu \mathrm{G} \mathrm{kpc}\right)$. Furthermore, the direction of $\vec{F}$ is obtained with a median error of $3.9^{\circ}$ and $5.8^{\circ}$ without and with experimental resolution respectively, while the position of the source is obtained with a median error of $0.2^{\circ}$ and $0.5^{\circ}$ without and with experimental uncertainty and applying a linear fit.

We found that the quadratic fit gives more accurate results than the linear fit when no measurement errors are introduced. However, for the magnitude of the experimental uncertainties considered, the linear fit is more accurate than the quadratic one when the experimental errors are taken into account. Only an experiment with very good energy and angular resolution (for example $0.3^{\circ}$ in angle and 5\% in energy) would have a better accuracy by using the quadratic fit instead of the linear one with 10 events from the same source. If more than 10 events above 30 $\mathrm{EeV}$ from a source were detected, the reconstruction accuracy is improved in a greater relative measure for the quadratic fit than for the linear one. For example, for 25 or more detected events, an angular resolution of $0.5^{\circ}$ and an energy resolution better than $10 \%$, the quadratic fit results become more accurate than the linear ones. In general, we can say that the quadratic fit is preferable whenever the value of the quadratic term is incompatible with zero within the error bar of the fit.

The turbulent component of the galactic magnetic field does not have a significant effect in the reconstruction accuracy, except for some sources near the galactic plane that have multiple images at higher energies than when considering only the regular component. At these energies the secondary images appear near the principal one when comparing to the experimental uncertainties considered here and the effect on the reconstruction accuracy is not large.

These results show that once several events from the same source are detected, it will be possible to reconstruct the source position with a good accuracy. It will also be possible to measure the integral of the orthogonal component of the magnetic field in the direction of the source. This will nicely complement the rotation measure observations that provide the integral of the parallel component of the magnetic field, giving hence a new insight into this open astrophysical problem.

\section{Acknowledgments}

This work is supported by ANPCyT (grant PICT 13562-03) and CONICET (grant PIP 5231). We thank P. L. Biermann and B. Baughman for comments.

\section{References}

[1] J. Abraham et al [The Pierre Auger Collaboration], Correlation of the highest energy cosmic rays with nearby extragalactic objects, Science 318 (2007) 938-943 [astro-ph/0711.2256].

[2] Y. Uchihori, M. Nagano, M. Takeda, M. Teshima, J. Lloyd-Evans, A.A. Watson, Cluster analysis of extremely high-energy cosmic rays in the northern sky, Astropart. Phys. 13 (2000) 151 astro-ph/9908193.

[3] T. Stanev, Ultra high energy cosmic rays and the large scale structure of the galactic magnetic field, Astrophys. J. 479 (1997) 290 astro-ph/9607086.

[4] G. Medina Tanco, E. De Gouveia dal Pino, J. Horvath, Deflection of ultra-high energy cosmic rays by the galactic magnetic field: from the sources to the detector, Astrophys. J. 479 (1998) L200 |astro-ph/9707041].

[5] D. Harari, S. Mollerach, E. Roulet, The toes of the ultra high energy cosmic ray spectrum, J. High Energy Phys. 08 (1999) 022 |astro-ph/9906309]. 
[6] P.G. Tinyakov, I.I. Tkachev, Tracing protons through the galactic magnetic field: A Clue for charge composition of ultrahigh-energy cosmic rays, Astropart. Phys. 18 (2002) 165 astro-ph/0111305].

[7] M. Prouza, R. Smida, The galactic magnetic field and propagation of ultrahigh energy cosmic rays, Astron. Astrophys. 410 (2003) 1 |astro-ph/0307165].

[8] H. Takami, K. Sato, Distortion of ultrahigh-energy sky by Galactic Magnetic Field, Astrophys. J. 681 (2008) 1279 [astro-ph/0711.2386].

[9] J. Alvarez-Muñiz, R. Engel, T. Stanev, Ultrahigh-energy cosmic ray propagation in the galaxy: Clustering versus isotropy, Astrophys. J. 572 (2001) 185 |astro-ph/0112227|.

[10] D. Harari, S. Mollerach, E. Roulet, Signatures of galactic magnetic lensing upon ultra high energy cosmic rays, J. High Energy Phys. 02 (2000) 035 [astro-ph/0001084].

[11] D. Harari, S. Mollerach, E. Roulet and F. Sanchez, Lensing of ultra high energy cosmic rays in turbulent magnetic fields, J. High Energy Phys. 03 (2002) 045 [astro-ph/0202362].

[12] D. Harari, S. Mollerach, E. Roulet, Astrophysical magnetic field reconstruction and spectroscopy with ultra high energy cosmic rays, J. High Energy Phys. 07 (2002) 006 [astro-ph/0205484].

[13] J. L. Han, Magnetic structure of our Galaxy: A review of observations, IAU Symp.259 'Cosmic Magnetic Fields: From Planets, to Stars and Galaxies' Proceedings (2009) [astro-ph/0901.1165].

[14] R. Beck, Galactic and extragalactic magnetic fields, astro-ph/0810.2923.

[15] H. Men, K. Ferriere and J. L. Han, Observational constraints on models for the interstellar magnetic field in the Galactic disk, (2008) [astro-ph/0805.3454].

[16] C.Heiles, The Local Direction and Curvature of the Galactic Magnetic Field Derived from Starlight Polarization, Astrophys. J. 462 (1996) 316.

[17] J. L. Han, R. N. Manchester, A. G. Lyne, G. J. Qiao and W. van Straten, Pulsar rotation measures and the largescale structure of Galactic magnetic field, Astrophys. J. 642 (2006) 868-881 [astro-ph/0601357].

[18] R. Beck, A. Shukurov, D. Sokoloff, R. Wielebinski, Systematic bias in interstellar magnetic field estimates, Astron. Astrophys. 411 (2003) 99-107 |astro-ph/0307330|

[19] R. Cowsik, J. Mitteldorf, Turbulence-enhanced synchrotron radiation in the Galaxy, Astrophys. J. 189 (1974) 51.

[20] S. Sarkar, Does the galactic synchrotron radio background originate in old supernova remnants?, Mon. Not. R. Astron. Soc. 199 (1982) 97.

[21] R. J. Rand, S.R. Kulkarni, The Local Galactic Magnetic Field, Astrophys. J. 343 (1989) 760-772.

[22] H. Ohno, S. Shibata, The Random Magnetic Field in the Galaxy, Mon. Not. R. Astron. Soc. 262 (1993) $953-962$.

[23] D. Harari, S. Mollerach, E. Roulet, Detecting filaments in the ultra high energy cosmic ray distribution, Astropart. Phys. 25 (2006) 412 [astro-ph/0602153]. 